\section{About the Materials} Research Society

The Materials Research Society (MRS), a not-forprofit scientific association founded in 1973, promotes interdisciplinary goal-oriented basic research on materials of technological importance. Membership in the Society includes over 12,000 scientists, engineers, and research managers from industrial, government, and university research laboratories in the United States and close to 70 countries.

The Society's interdisciplinary approach differs from that of single-discipline professional societies because it promotes information exchange across the many technical fields touching materials development. MRS sponsors two major international annual meetings encompassing approximately 70 topical symposia, and also sponsors numerous single-topic scientific meetings. The Society recognizes professional and technical excellence and fosters technical interaction in local geographic regions through Sections and University Chapters.

MRS participates in the international arena of materials research through the International Union of Materials Research Societies (IUMRS). MRS is a member of the Federation of Materials Societies and is an affiliate of the American Institute of Physics.

MRS publishes symposium proceedings, $M R S$ Bulletin, Journal of Materials Research, and other publications related to current research activities.

MRS Bulletin (ISSN: 0883-7694) is published 12 times a year by the Materials Research Society, 506 Keystone Drive, Warrendale, PA, 15086-7573. Periodical postage paid at Warrendale, PA and at additional mailing offices. POSTMASTER: Send address changes to MRS Bulletin in care of the Materials Research Society, at the address listed; tel. 724-7793003; fax 724-779-8313. Printed in the U.S.A.

Membership in MRS is $\$ 100$ annually for regular members, \$25 for students. Dues include an allocation of $\$ 29$ (\$17 for students) to a subscription to MRS Bulletin. Individual member subscriptions are for personal use only. Non-member subscription rates are $\$ 190$ for one calendar year (12 issues) within the U.S.A. and \$245 elsewhere. Single copies may be purchased for $\$ 20$ each. Requests from subscribers for missing journal issues will be honored without charge only if received within six months of the issue's actual date of publication; otherwise, the issue may be purchased at the single-copy price.

MRS Bulletin is included in Current Contents ${ }^{\otimes}$ Engineering, Computing, and Technology; Current Contents ${ }^{\circledast}$ Physical, Chemical, and Earth Sciences, the SciSearch ${ }^{\circledast}$ online database, Research Alert ${ }^{\circledast}$, Science Citation Index ${ }^{\oplus}$, and the Materials Science Citation Index ${ }^{\text {Tnu }}$. Back volumes of MRS Bulletin are available in $16 \mathrm{~mm}$ microfilm, $35 \mathrm{~mm}$ microfilm, or $105 \mathrm{~mm}$ microfiche through University Microfilms Inc., 300 North Zeeb Road, Ann Arbor, Michigan 48106, USA.

Send Letters to the Editor to:

MRS Bulletin

Materials Research Society

506 Keystone Drive

Warrendale, PA 15086-7573 USA

Fax 724-779-8313

Bulletin@mrs.org

Letters must include your name, affiliation, and full contact information.

www.mrs.org/publications/bulletin

\title{
M|RIS BULLETIN
}

Editorial Office • 506 Keystone Drive • Warrendale, PA 15086-7573 USA

Tel. 724-779-3004 ext. 522; fax 724-779-8313; Bulletin@mrs.org; http://www.mrs.org/

Editor

E.L. Fleischer

Managing Editor

J. Meiksin

Technical Editor

A. Bertram Wilson

Art Director

E. Stiller

Design/Production

S. Franklin and H. Miller

Editorial Assistants

S.A. Novosel and M. Wilmoth

Advertising

M.E. Kaufold and D.L. Watterson
Circulation

S. Forrest

Guest Editors

H. Hillmer and R. Germann

Special Contributors

E. Jarvis, G. Khitrov, D. Litvinov, C. Oertel,

G. Rao, A. Reller, P. Siffert, B. Stritzker, and S. Trohalak

Special Consultants

M.-I. Baraton and T. Besmann

Associate Technical Editor

T. Palucka
Associate Editor-Europe

I.W. Boyd, University College London

Dept. of Electronic and

Electrical Engineering

Torrington Place

London WCI E7 JE, UK

Tel. 44-171-380-7300 or 7302

Book Review Board

J.M. Phillips (Chair), R.W. Cahn,

M. Coltrin, J. Curro, G. Kellogg,

G.G. Long, and L. Olafsen

MRS Office of Public Affairs

499 South Capitol Street SW, Suite 420, Washington DC 20003

Tel. 202-256-5211

\section{EDITORIAL BOARD}

A.L. Greer, Chair

Cambridge University

Cambridge, UK

V.S. Arunachalam

Carnegie Mellon University

Pittsburgh, PA

E. Arzt

Max-Planck-Institut

für Metallforschung and

University of Stuttgart

Stuttgart, Germany

R.W. Cahn

Cambridge University

Cambridge, UK

\section{VOLUME ORGANIZERS}

2002

R.W. Cahn

Cambridge University

Cambridge, UK

2003

L.A. Clevenger

IBM Microelectronics

Hopewell Junction, NY

2004

P.S. Drzaic

Alien Technology Corp.

Morgan Hill, CA
D.J. Eaglesham

Lawrence Livermore National Laboratory Arizona State University

Livermore, CA

E.J. Kramer

University of California-

Santa Barbara

Santa Barbara, CA

G.G. Long

National Institute of Standards

and Technology

Gaithersburg, MD

S.C. Moss

Aerospace Corporation

Los Angeles, CA
S.T. Picraux

Tempe, AZ

Y. Shiohara

ISTEC

Tokyo, Japan

C.C. Tsai

Quanta Display Inc.

Taiwan

J.H. Westbrook

Brookline Technologies

Ballston Spa, NY

\section{MRS OFFICERS}

\begin{tabular}{lll}
\hline President & Secretary & Immediate Past President \\
M.J. Mayo & A.J. Hurd & A.H. King \\
GUIRR, National Research Council & Los Alamos National Laboratory & Purdue University \\
Vice President and President-Elect & Treasurer & Executive Director \\
H.E. Katz & L. Horton & Materials Research Society \\
Bell Labs/Lucent Technologies & Oak Ridge National Laboratory & J.B. Ballance
\end{tabular}

\section{INTERNATIONAL UNION OF MATERIALS RESEARCH SOCIETIES}

\begin{tabular}{|c|c|c|c|c|c|c|c|}
\hline $\begin{array}{l}\text { President } \\
\text { R.J. Nemanich } \\
\text { North Carolina State Univ. } \\
\text { Raleigh, NC, USA }\end{array}$ & $\begin{array}{l}\text { First Vice President } \\
\text { L. Zhou } \\
\text { NIN } \\
\text { Xian, Shaanxi, China }\end{array}$ & \multicolumn{2}{|c|}{$\begin{array}{l}\text { Second Vice President } \\
\text { P. Rama Rao } \\
\text { University of Hyderabad } \\
\text { Hyderabad, India }\end{array}$} & $\begin{array}{l}\text { Secretary } \\
\text { M.J. Yacamán } \\
\text { University of Texas } \\
\text { at Austin } \\
\text { Austin, TX, USA }\end{array}$ & $\begin{array}{l}\text { Treasurer } \\
\text { J.-W. Park } \\
\text { Hanyang University } \\
\text { Seoul, South Korea }\end{array}$ & $\begin{array}{l}\text { Immediate Past President } \\
\text { P.A. Glasow } \\
\text { Erlangen, Germany }\end{array}$ & $\begin{array}{l}\text { General Secretary } \\
\text { R.P.H. Chang } \\
\text { Northwestern University } \\
\text { Evanston, IL, USA }\end{array}$ \\
\hline \multicolumn{3}{|c|}{$\begin{array}{l}\text { Australian Materials Research Society (A-MRS) } \\
\text { J.S. Williams, Australian National University }\end{array}$} & \multicolumn{3}{|c|}{$\begin{array}{l}\text { Materials Research Society of Argentina (MRS-Argentina) } \\
\text { R. Romero, IFIMAT }\end{array}$} & \multicolumn{2}{|c|}{$\begin{array}{l}\text { Materials Research Society of Russia (MRS-Russia) } \\
\text { N.Z. Lyakhov, Russian Academy of Sciences }\end{array}$} \\
\hline \multicolumn{3}{|c|}{$\begin{array}{l}\text { Chinese Materials Research Society (C-MRS) } \\
\text { L. Zhou, NW Institute for Nonferrous Metal Research }\end{array}$} & \multicolumn{3}{|c|}{$\begin{array}{l}\text { Materials Research Society of India (MRS-I) } \\
\text { D. Chakravorty, Indian Association for the Cultivation of Science }\end{array}$} & \multicolumn{2}{|c|}{$\begin{array}{l}\text { Materials Research Society of Singapore (MRS-S) } \\
\text { B.V.R. Chowdari, National University of Singapore }\end{array}$} \\
\hline \multicolumn{3}{|c|}{$\begin{array}{l}\text { European Materials Research Society (E-MRS) } \\
\text { G. Marletta, Univerita degli Studi di Catania }\end{array}$} & \multicolumn{3}{|c|}{$\begin{array}{l}\text { Materials Research Society of Japan (MRS-J) } \\
\text { T. Kishi, National Institute for Materials Science }\end{array}$} & \multicolumn{2}{|c|}{$\begin{array}{l}\text { Materials Research Society of Taiwan (MRS-T) } \\
\text { L.J. Chen, National Tsing Hua University }\end{array}$} \\
\hline \multicolumn{3}{|c|}{$\begin{array}{l}\text { Materials Research Society (MRS) } \\
\text { M.J. Mayo, GUIRR, National Research Council }\end{array}$} & \multicolumn{3}{|c|}{$\begin{array}{l}\text { Materials Research Society of Korea (MRS-K) } \\
\text { S.-G. Kang, Hanyang University }\end{array}$} & \multicolumn{2}{|c|}{$\begin{array}{l}\text { Mexican Materials Research Society (Mexican-MRS) } \\
\text { V. Rodríguez Lugo, Benemérita Universidad Autónoma de Puebla }\end{array}$} \\
\hline
\end{tabular}

